\title{
Active lifestyles related to excellent self-rated health and quality of life: cross sectional findings from 194,545 participants in The 45 and Up Study
}

\author{
Richard R Rosenkranz ${ }^{1,2^{*}}$, Mitch J Duncan ${ }^{3}$, Sara K Rosenkranz ${ }^{2}$ and Gregory S Kolt ${ }^{1}$
}

\begin{abstract}
Background: Physical activity and sitting time independently contribute to chronic disease risk, though little work has focused on aspirational health outcomes. The purpose of this study was to examine associations between physical activity, sitting time, and excellent overall health (ExH) and quality of life (ExQoL) in Australian adults.

Methods: The 45 and Up Study is a large Australian prospective cohort study ( $n=267,153)$. Present analyses are from 194,545 participants (48\% male; mean age $=61.6 \pm 10.7 \mathrm{yrs}$ ) with complete baseline questionnaire data on exposures, outcomes, and potential confounders (age, income, education, smoking, marital status, weight status, sex, residential remoteness and economic advantage, functional limitation and chronic disease). The Active Australia survey was used to assess walking, moderate, and vigorous physical activity. Sitting time was determined by asking participants to indicate number of hours per day usually spent sitting. Participants reported overall health and quality of life, using a five-point scale (excellent-poor). Binary logistic regression models were used to analyze associations, controlling for potential confounders.
\end{abstract}

Results: Approximately $16.5 \%$ of participants reported ExH, and $25.7 \%$ reported ExQoL. In fully adjusted models, physical activity was positively associated with $\mathrm{ExH}$ ( $\mathrm{AOR}=$ adjusted odds ratio for most versus least active $=2.22$, $95 \% \mathrm{Cl}=2.20,2.47 ; P_{\text {trend }}<0.001$ ) and ExQoL (AOR for most versus least active $=2.30,95 \% \mathrm{Cl}=2.12,2.49 ; P_{\text {trend }}<$ 0.001). In fully adjusted models, sitting time was inversely associated with ExH (AOR for least versus most sitting group $=1.13,95 \% \mathrm{Cl}=1.09,1.18 ; P_{\text {trend }}<0.001$ ) and ExQoL (AOR for least versus most sitting group $=1.13,95 \%$ $\left.\mathrm{Cl}=1.10,1.17 ; P_{\text {trend }}<0.001\right)$. In fully adjusted models, interactions between physical activity and sitting time were not significant for ExH $(P=0.118)$ or ExQoL $(P=0.296)$.

Conclusions: Physical activity and sitting time are independently associated with excellent health and quality of life in this large diverse sample of Australian middle-aged and older adults. These findings bolster evidence informing health promotion efforts to increase PA and decrease sitting time toward the achievement of better population health and the pursuit of successful aging.

Keywords: Physical activity, Sedentary behavior, Sitting time, Sedentary lifestyle, Salutogenic, Health promotion, Adult, Older adult, Successful aging, Quality of life

\footnotetext{
* Correspondence: ricardo@ksu.edu

${ }^{1}$ School of Science and Health, University of Western Sydney, Sydney, Australia ${ }^{2}$ Department of Human Nutrition, Kansas State University, Manhattan, KS, USA Full list of author information is available at the end of the article
} 


\section{Background}

Worldwide, nations are preparing for the demands of an aging population, and this entails dealing with challenges of maintaining health, functional capacity, and wellbeing [1]. Focusing on relevant lifestyle behaviors is an important consideration for preventing or delaying chronic disease and improving health [1-3]. An emerging body of literature indicates that the lifestyle behaviors of physical activity and time spent sitting independently contribute to health outcomes such as chronic disease morbidity and mortality risk [4].

Regularly engaging in moderate-to-vigorous physical activity has been shown to reduce the risk of all-cause mortality, cardiovascular mortality, cancer mortality, stroke, heart disease, breast cancer, colon cancer, and other undesirable health outcomes [5]. Over the past decade, however, research on the health impacts of sedentary behavior (time spent at low levels of energy expenditure while in a sitting posture) has expanded rapidly [4]. High volumes of time spent sitting are associated with an increased risk of all-cause mortality [6-10], cardiovascular disease mortality [8], type 2 diabetes mellitus [11-15], and other diseases or conditions [15-17] when adjusting for participation in moderate-tovigorous intensity physical activity. Therefore, insufficient moderate-to-vigorous physical activity and sitting time may be distinct influences on poor health.

Compared to abundant literature on risk factors for disease and poor health, research focusing on the influence of physical activity and sitting time on more aspirational health-related outcomes is much less common [5,18-20]. Successful aging has been described as a multidimensional intersection, where not only the avoidance of disease and disability are found, but also where high cognitive and physical function and engagement with life conjoin [21]. The focus on such aspirational outcomes represents a "salutogenic" approach to health promotion [22], rather than the traditional disease prevention approach. This salutogenic orientation is instructive for determining influences on aspirational levels of health and well-being. Aspirational positively framed messages may be more effective for motivating healthful behavior in some segments of the population, compared to focusing on the avoidance of chronic disease, which is often an abstract possibility many years away [23].

Despite the aging population and widespread problem of physical inactivity, there has been limited use of successful aging or salutogenic approaches to frame positive health messages toward motivating active lifestyles. This study examines both self-reported health and quality of life status as they are useful health outcomes and are predictive of more objective health indicators [24]. To investigate whether higher levels of physical activity and lower levels of sitting time were positively associated with excellent health and quality of life, we utilized selfreported data from a large sample of middle-aged and older Australian men and women, and we statistically adjusted for a range of associated covariates and potential confounding variables in the analyses.

\section{Methods}

\section{The 45 and Up Study}

The 45 and Up Study is a large ongoing Australian prospective cohort study that began with a baseline sample of 267,153 men and women from New South Wales, the most populous state in Australia. A detailed description of The 45 and Up Study has been published previously [25]. The 45 and Up Study baseline [26] data provide information on a wide range of health-related variables. Participants were randomly sampled from the Medicare Australia (national health insurance) database between February 2006 and December 2008. All adults who were aged 45 years and over and who were currently residing in NSW at the time of recruitment were eligible for inclusion in the Study. Participants who completed a mailed baseline questionnaire and provided their signed consent for participation in the baseline questionnaire and long-term follow-up were included in the Study [25]. The University of NSW Human Research Ethics Committee provided approval for The 45 and Up Study and analysis of the baseline questionnaire data (approval number 05035). The University of Western Sydney Human Research Ethics Committee granted reciprocal institutional ethics approval for use of the baseline questionnaire data in the current study (UWS Protocol number H8793).

\section{Participants}

Participants were a subgroup $(n=194,545)$ of the total baseline sample of 267,153 men and women enrolled in The 45 and Up Study as of December 2009 (18\% response rate). The 45 and Up Study sample was intended to be a large heterogeneous sample of Australian adults, though not necessarily a true representation of the Australian adult population. The present study's sample included all participants aged 45-106 years with nonmissing data on self-rated overall health, quality of life, physical activity, sitting time, and covariates and potential confounding variables (age, household income, educational qualification, smoking status, marital status, weight status, sex, residential remoteness and economic advantage, functional limitation, and number of chronic diseases). Thus, the final sample included 194,545 residents (48\% male) of New South Wales, aged 45106 years (mean $\pm \mathrm{SD}=61.6 \pm 10.7$ yrs), from The 45 and Up Study baseline dataset. All participant data, except region of residence (Medicare records), originated 
from responses to a self-administered paper questionnaire that was completed and returned by postal mail.

\section{Physical activity and sitting time}

The Active Australia Survey (AAS) [27], was used to measure physical activity in The 45 and Up Study baseline questionnaire. This instrument has previously demonstrated acceptable test-retest reliability [28] and validity [29]. On the questionnaire, participants were asked to indicate their participation in three types of physical activity over the previous week- "walking continuously, for at least 10 minutes (for recreation or exercise or to get to or from places)"; "vigorous physical activity (that made you breathe harder or puff and pant, like jogging, cycling, aerobics, competitive tennis, but not household chores or gardening)"; and "moderate physical activity (like gentle swimming, social tennis, vigorous gardening or work around the house)" - by recording the total duration and the total number of times they participated in each [27]. For this study, total minutes spent in the queried physical activities was used to determine physical activity levels, with vigorous physical activity time multiplied by two, for double weighting [27]. In accordance with previous research using this dataset [15] physical activity time was divided into five categories of total minutes per week, as follows: zero mins; low active (1-149 mins); sufficiently active (150299 mins); highly active (300-539 mins); and very highly active $(540+$ mins $)$.

Total sitting time was determined by asking participants to report total hours per day usually spent sitting. In accordance with previous research arising from the 45 and Up baseline dataset [15], sitting time was divided into four categories of 0 to $<4$ hours; 4 to $<6$ hours; 6 to $<8$ hours; and 8 hours or more of sitting time per day for analysis. Although reliability and validity of this sitting time questionnaire item has not been formally assessed, the item is analogous to the sitting time item used in the International Physical Activity Questionnaire (IPAQ), shown to have acceptable reliability and validity [30]. Atkin et al. [31] support the use of single-item questionnaires in epidemiological research, when the primary requirements of such research consist of the ability to rank levels of health-related variables within the sample.

\section{Self-rated health and quality of life}

Self-rated overall health was assessed with the following question, "In general, how would you rate your overall health?" Five response options included: excellent; very good; good; fair; or poor. For the current study, selfrated health was dichotomized as excellent or not excellent (including very good; good; fair; and poor). Self-rated quality of life was assessed with the following question, "In general, how would you rate your quality of life?" Five response options included: excellent, very good, good, fair, or poor. Self-rated quality of life was dichotomized as excellent or not excellent.

\section{Covariates and potential confounding variables}

To control potential confounding in analyses, covariates included age group, household income, educational qualification, residential remoteness, residential economic advantage, marital status, smoking status, weight status, number of chronic diseases, and level of functional limitation. Participants indicated their age in years, categorized into five age groups: 45 to $54 ; 55$ to $64 ; 65$ to $74 ; 75$ to $84 ; 85$ and up. Highest educational qualification was categorically self-reported, including: no school certificate; school certificate; high school certificate; trade or apprenticeship; certificate or diploma; or university degree. Participants indicated whether they had ever been a regular smoker; smoking status was dichotomously categorized as "ever" or "never."

Marital status was self-reported according to six categories, reduced to a dichotomous variable for analysis as married (including married; de facto/living with a partner) or not married (single; widowed; divorced; separated). Residential remoteness and residential economic advantage were determined based on the mean Accessibility Remoteness of Australia Plus score for participant home address postcode. Five residential remoteness categories included: major city, inner regional, outer regional, remote, or very remote area. Four residential economic advantage categories included: least, mid to low, mid to high, and most economic advantage. Annual household income was categorized for analysis as less than $\$ 10,000, \$ 10,000-\$ 29,999, \$ 30,000-\$ 49,999$, $\$ 50,000-\$ 69,999$, or $\$ 70,000$ or more.

Weight status was determined from self-reported height and weight to calculate body mass index $\left(\mathrm{km} / \mathrm{m}^{2}\right)$, using WHO classifications [32] to determine underweight $\left(<18.50 \mathrm{~km} / \mathrm{m}^{2}\right)$, normal weight $\left(18.50-24.99 \mathrm{~km} / \mathrm{m}^{2}\right)$, overweight $\left(25.00-29.99 \mathrm{~km} / \mathrm{m}^{2}\right)$ and obese $\left(\geq 30.00 \mathrm{~km} / \mathrm{m}^{2}\right)$ categories. Functional limitation status was determined using the Medical Outcomes Study Physical Functioning (MOS-PF) scale, which assesses the extent to which an individuals' health limits their ability to perform daily functional activities [33]. The MOS-PF has demonstrated good test-retest reliability and content validity as a measure of physical functioning [34]. Based on a 100-point scale, functional limitation scores were categorized as: no functional limitation (100), minor limitation (95-99), moderate limitation (85-94), or severe limitation (0-84).

Participants reported whether they had ever been told by a doctor that they have skin cancer, melanoma, breast cancer, other cancer, heart disease, prostate cancer, enlarged prostate, high blood pressure, stroke, diabetes, 
blood clot, asthma, hay fever, depression, anxiety, or Parkinson's disease. Chronic diseases were categorized for analysis as: none, one, or two or more chronic diseases.

\section{Statistical methods}

Data from The 45 and Up Study baseline dataset were analyzed using SPSS 19.0 software (SPSS Inc. Chicago, IL USA) for both descriptive and inferential statistics. Crude odds ratios (OR) and adjusted odds ratios (AOR) with $95 \%$ confidence intervals $(\mathrm{CI})$ were calculated to assess the association between exposures and the outcome variables of excellent health and excellent quality of life using separate binary logistic regression models. Potential confounders were added to the model in groups of demographic and physical health variables, and interaction between physical activity and sitting time was examined via an interaction term. Logistic regression models were mutually adjusted for categories of physical activity or sitting time (model 1); followed by additional adjustment for categories of age, household income, educational qualification, smoking status, marital status, weight status, sex, and remoteness and economic advantage of residential area (model 2); and lastly, a fully adjusted model included additional adjustment for categories of physical limitation and chronic diseases (model 3). To examine the consistency of relationships between active lifestyle variables and health-related outcomes, follow-up logistic regression analyses were used, stratified by age group, sex, household income, weight status, and self-reported ancestry (Australian or not). A final fully adjusted binary logistic regression (model 3) was used to examine excellent self-rated health and quality of life by 20 combination categories of five physical activity and four sitting levels. A significance level of alpha $=0.05$ was used for all analyses.

\section{Results}

Approximately $16.5 \%$ of the sample reported excellent overall health and $25.7 \%$ reported excellent quality of life (Table 1). The unadjusted associations between sociodemographic and lifestyle factors are provided in Table 2. For both excellent quality of life and excellent health, significant bivariate relationships were found for sitting time (inverse), physical activity, sex, marital status, age (inverse), income, education, residential economic advantage, residential remoteness (inverse), smoking (inverse), chronic disease (inverse), functional limitation, (inverse) and weight status (inverse from normal weight).

\section{Sitting time}

Table 3 presents the odds of excellent overall self-rated health and excellent quality of life by categories of sitting time. In model 1 , the lowest sitting time category showed a significant positive association in log-odds of
Table 1 Self-rated health and quality of life status prevalence and active lifestyles in the $\mathbf{4 5}$ and Up Study baseline sample $(\mathrm{N}=194,545)$

\begin{tabular}{|c|c|c|c|c|c|c|}
\hline & \multicolumn{2}{|c|}{$\begin{array}{c}\text { Total } \\
\text { sample }\end{array}$} & \multicolumn{2}{|c|}{$\frac{\text { Very highly active }^{\#}}{\geq 540 \mathrm{mins} / \text { week }}$} & \multicolumn{2}{|c|}{$\frac{\text { Sitting }^{\ddagger}}{0 \text { to }<4 \text { hrs/day }}$} \\
\hline & $\mathbf{N}$ & $\%$ & $\mathrm{~N}$ & $\%$ & $\mathbf{N}$ & $\%$ \\
\hline \multicolumn{7}{|l|}{ Self-rated health } \\
\hline Excellent & 31,738 & 16.5 & 17,763 & 56.0 & 9,214 & 29.0 \\
\hline Very Good & 73,852 & 38.4 & 35,158 & 47.6 & 19,494 & 26.4 \\
\hline Good & 62,417 & 32.5 & 24,127 & 38.7 & 15,373 & 24.6 \\
\hline Fair & 20,629 & 10.7 & 6,201 & 30.1 & 4,452 & 21.6 \\
\hline Poor & 3,672 & 1.9 & 723 & 19.7 & 698 & 19.0 \\
\hline \multicolumn{7}{|c|}{ Self-rated quality of life } \\
\hline Excellent & 48,787 & 25.7 & 25,679 & 52.6 & 13,531 & 27.7 \\
\hline Very Good & 73,141 & 38.5 & 32,775 & 44.8 & 18,597 & 25.4 \\
\hline Good & 50,328 & 26.5 & 19,260 & 38.3 & 12,498 & 24.8 \\
\hline Fair & 14,929 & 7.9 & 4,573 & 30.6 & 3,358 & 22.5 \\
\hline Poor & 2,792 & 1.5 & 677 & 24.2 & 563 & 20.2 \\
\hline
\end{tabular}

"Refers to those within each level of health and quality of life who were very highly active;

${ }^{\ddagger}$ Refers to those within each level of health and quality of life who sat 0 to $<4 \mathrm{hrs} /$ day.

excellent health and quality of life, relative to the highest sitting time category of $\geq 8$ hours per day (AOR for lowest versus highest category $=1.09 ; 95 \% \mathrm{CI}=1.05,1.13$; $\left.P_{\text {trend }}<0.001\right)$ and excellent quality of life (AOR for lowest versus highest category $=1.07 ; 95 \% \mathrm{CI}=1.04,1.10$; $\left.P_{\text {trend }}<0.001\right)$.

In the fully adjusted model 3, all categories of sitting time displayed significantly higher log-odds of excellent self-rated health, relative to the category sitting $\geq 8$ hours per day. The category reporting the lowest amount of sitting was $13 \%$ more likely to report excellent health (AOR for lowest versus highest category $=1.13 ; 95 \% \mathrm{CI}=1.09$, 1.18; $\left.P_{\text {trend }}<0.001\right)$ compared to those sitting $\geq 8$ hours per day. All categories of sitting time showed higher logodds of excellent quality of life, compared with the highest sitting category. The category reporting least sitting time was $13 \%$ more likely to report excellent quality of life (AOR for lowest versus highest category $=1.13$; 95\% $\left.\mathrm{CI}=1.10,1.17 ; P_{\text {trend }}<0.001\right)$ compared to those sitting $\geq 8$ hours per day.

\section{Physical activity}

Table 3 presents the odds of excellent overall health and excellent quality of life by five categories of physical activity. In model 1, all categories of physical activity above zero minutes showed significantly higher log-odds of excellent health and quality of life, relative to those reporting zero minutes. The most physically active group was more than four times as likely to report excellent health (AOR for highest versus lowest category $=4.51 ; 95 \% \mathrm{CI}=4.08$, 
Table 2 Bivariate associations between participant characteristics and self-rated health and quality of life status in the 45 and Up Study baseline sample $(N=194,545)$

\begin{tabular}{|c|c|c|c|c|}
\hline & & & Excellent health & Excellent quality of lif \\
\hline & $\mathrm{N}$ & $\%$ & $\mathrm{OR}^{*}(95 \% \mathrm{Cl})$ & $\mathrm{OR}^{*}(95 \% \mathrm{Cl})$ \\
\hline \multicolumn{5}{|l|}{ Sex } \\
\hline Malet & 95,242 & 48.4 & 1.00 & 1.00 \\
\hline Female & 100,293 & 51.6 & $1.38(1.34-1.41)$ & $1.31(1.28-1.33)$ \\
\hline
\end{tabular}

Sitting time per day (hours)

$\geq 8+$
6 to $<8$
4 to $<6$
0 to $<4$

Physical activity per week (minutes)

$$
\begin{aligned}
& \text { 0† } \\
& 1 \text { to } 149 \\
& 150 \text { to } 299 \\
& 300 \text { to } 539 \\
& \geq 540
\end{aligned}
$$

50,402

38,806

55,435

49,902

7,624

30,086

30,524

41,413

84,898

Age group (years)

$$
\begin{aligned}
& 45 \text { to } 54 \dagger \\
& 55 \text { to } 64 \\
& 65 \text { to } 74 \\
& 75 \text { to } 84 \\
& 85 \text { and older }
\end{aligned}
$$

62,038

65,796

40,444

22,037

4,230

Household income

$$
\begin{aligned}
& \text { less than } \$ 10 \mathrm{~K} \dagger \\
& \$ 10 \text { to }<30 \mathrm{~K} \\
& \$ 30 \text { to }<50 \mathrm{~K}^{\#} \\
& \$ 50 \text { to }<70 \mathrm{~K} \\
& \$ 70 \mathrm{~K} \text { or more }
\end{aligned}
$$

Residential remoteness

$$
\begin{aligned}
& \text { Major city† } \\
& \text { Inner regional area } \\
& \text { Outer regional area } \\
& \text { Remote area } \\
& \text { Very remote area }
\end{aligned}
$$

Residential economic advantage

$$
\begin{aligned}
& \text { Least advantaged }+ \\
& \text { Mid-low advantage } \\
& \text { Mid-high advantage } \\
& \text { Most advantaged }
\end{aligned}
$$

Educational qualification

$$
\begin{aligned}
& \text { No school certificate } \\
& \text { School certificate } \\
& \text { High school certificate } \\
& \text { Trade/apprenticeship }
\end{aligned}
$$

$$
1.00
$$

$$
\begin{aligned}
& 1.11(1.07-1.15) \\
& 1.25(1.20-1.29)
\end{aligned}
$$$$
1.68(1.62-1.74)
$$

$1.44(1.34-1.53)$

10.1

11.0

\subsection{0}

$1.03(0.99-1.06)$

$1.05(1.02-1.08)$

$1.18(1.15-1.22)$

1.00

$1.75(1.61-1.90)$

$2.65(2.44-2.87)$

$3.34(3.09-3.61)$

4.05 (3.75-4.38)

\subsection{0}

$0.94(0.92-0.97)$

$0.69(0.67-0.71)$

$0.37(0.35-0.39)$

$0.21(0.19-0.23)$

\subsection{0}

$1.33(1.25-1.43)$

$2.38(2.23-2.53)$

$2.93(2.74-3.14)$

$4.37(4.09-4.66)$

1.00

$1.02(1.00-1.04)$ $0.98(0.95-1.01)$

$0.88(0.81-0.96)$

$0.96(0.74-1.25)$

1.00

$1.07(1.04-1.10)$

$1.15(1.12-1.18)$

$1.47(1.43-1.51)$
1.00

$1.92(1.82-2.03)$

$1.50(1.42-1.58)$
$1.57(1.50-1.65)$ 
Table 2 Bivariate associations between participant characteristics and self-rated health and quality of life status in the 45 and Up Study baseline sample ( $\mathrm{N}=194,545)$ (Continued)

\section{Certificate/diploma \\ University degree}

Smoking status

Never regular smokert

Ever regular smoker

Weight status

Underweight
Normal weight†
Overweight
Obese

Chronic diseases

Nonet

One

Two or more

Functional limitation

No limitationt
Minor limitation
Moderate limitation
Severe limitation

Marital status

\section{3,251 \\ 50,949}

110,119

84,426

2,404

71,499

77,684

42,958

71,221

67,886

55,438

66,747

34,012

36,819

56,967

45,492

149,053

\section{2}

26.2

56.6

43.4

1.2

36.8

39.9

22.1

36.6

34.9

28.5

34.3

17.5

18.9

29.3

23.4

76.6
$2.20(2.08-2.33)$

$3.34(3.16-3.53)$

1.00

$0.67(0.65-0.68)$

$0.65(0.58-0.72)$

1.00

$0.59(0.57-0.60)$

$0.24(0.23-0.25)$

1.00

$0.59(0.57-0.60)$

$0.23(0.22-0.24)$

1.00

$0.40(0.38-0.41)$

$0.20(0.19-0.20)$

$0.05(0.05-0.06)$

1.00

$1.33(1.29-1.37)$
$2.22(2.12-2.33)$

$3.29(3.14-3.44)$

1.00

$0.72(0.71-0.74)$

$0.59(0.53-0.65)$

1.00

$0.76(0.74-0.78)$

$0.46(0.45-0.48)$

1.00

$0.75(0.73-0.76)$

$0.43(0.42-0.44)$

1.00

$0.64(0.62-0.65)$

$0.39(0.38-0.40)$

$0.12(0.11-0.12)$

1.00

$1.82(1.77-1.86)$

†Reference category; *Unadjusted odds ratio; \#includes those selecting "prefer not to answer".

4.98; $P_{\text {trend }}<0.001$ ) and excellent quality of life (AOR for highest versus lowest category $=4.05 ; 95 \% \mathrm{CI}=3.75,4.38$; $P_{\text {trend }}<0.001$ ), compared to the least physically active.

In the fully adjusted model 3 , all categories of physical activity above zero minutes displayed significantly higher log-odds of excellent health and quality of life, relative to the lowest physical activity category. The most physically active category was twice as likely to report excellent health (AOR for highest versus lowest category $=2.22$; $\left.95 \% \mathrm{CI}=2.00,2.47 ; P_{\text {trend }}<0.001\right)$ and twice as likely to report excellent quality of life (AOR for highest versus lowest category $=2.30 ; 95 \% \quad \mathrm{CI}=2.12,2.47 ; \quad P_{\text {trend }}<$ 0.001 ), compared to the least physically active.

\section{Stratified analyses}

A series of fully adjusted binary logistic regressions (model 3), used to examine variations across demographic variables, are presented in Additional file 1: Tables S1-S4. In age-stratified analyses, the relationship between physical activity and excellent health was strongest for the oldest age group (AOR for highest versus lowest category $=4.54 ; 95 \% \mathrm{CI}=1.78,11.56$ ). For the weight status-stratified analyses, the relationship between physical activity and excellent health was strongest for the underweight group (AOR for highest versus lowest category $=6.60 ; 95 \% C I=1.56,28.01$ ). For both health and quality of life, across all other strata of age, sex, household income, ancestry, and weight status, adjusted odds ratios for most physically active versus least active category centered just over 2.0, ranging from 1.58-2.80. For sitting time, adjusted odds ratios for lowest sitting time versus highest centered just over 1.0, ranging from $0.88-1.31$ for both health and quality of life outcomes, across all other strata.

\section{Interaction of physical activity and sitting time}

In logistic regression model 1 , examining relationships between physical activity, sitting time, and excellent health and quality of life, the physical activity and sitting time interaction terms were significant $(P=0.001$ for health; $P=0.003$ for quality of life). These interactions were not significant, however, in the fully adjusted models ( $P=0.118$ for health; $P=0.296$ for quality of life).

Figure 1 graphically displays the fully adjusted (model 3) log-odds of excellent health by 20 combinations of physical activity and sitting time. In this figure, the reference category is the most inactive group: those reporting zero minutes of physical activity and eight or more 
Table 3 Odds of excellent overall health and quality of life by sitting time and physical activity $(\mathrm{N}=194,545)$

\begin{tabular}{|c|c|c|c|}
\hline & \multicolumn{3}{|c|}{ Excellent health } \\
\hline & Adjusted $\mathrm{OR}^{\mathrm{a}}(95 \% \mathrm{Cl})$ & Adjusted $\mathrm{OR}^{\mathrm{b}}(95 \% \mathrm{Cl})$ & Adjusted $\mathrm{OR}^{\mathrm{c}}(95 \% \mathrm{Cl})$ \\
\hline \multicolumn{4}{|c|}{ Sitting time (hrs/day) } \\
\hline$\geq 8+$ & 1.00 & 1.00 & 1.00 \\
\hline 6 to $<8$ & $0.90(0.87-0.94)$ & $1.05(1.01-1.09)$ & $1.04(0.99-1.08)$ \\
\hline 4 to $<6$ & $0.90(0.87-0.93)$ & $1.10(1.06-1.14)$ & $1.05(1.01-1.09)$ \\
\hline 0 to $<4$ & $1.09(1.05-1.13)$ & $1.26(1.21-1.30)$ & $1.13(1.09-1.18)$ \\
\hline \multicolumn{4}{|c|}{ Physical activity (mins/week) } \\
\hline $0+$ & 1.00 & 1.00 & 1.00 \\
\hline 1 to 149 & $1.61(1.44-1.78)$ & $1.28(1.15-1.42)$ & $1.10(0.98-1.23)$ \\
\hline 150 to 299 & $2.53(2.28-2.81)$ & $1.73(1.56-1.92)$ & $1.37(1.23-1.53)$ \\
\hline 300 to 539 & $3.47(3.14-3.84)$ & $2.28(2.06-2.53)$ & $1.69(1.51-1.88)$ \\
\hline$\geq 540$ & $4.51(4.08-4.98)$ & $3.20(2.89-3.54)$ & $2.22(2.00-2.47)$ \\
\hline \multicolumn{4}{|c|}{ Excellent quality of life } \\
\hline & Adjusted $\mathrm{OR}^{\mathrm{a}}(95 \% \mathrm{Cl})$ & Adjusted OR ${ }^{b}(95 \% \mathrm{Cl})$ & Adjusted OR ${ }^{\mathrm{c}}(95 \% \mathrm{Cl})$ \\
\hline \multicolumn{4}{|c|}{ Sitting time (hrs/day) } \\
\hline$\geq 8+$ & 1.00 & 1.00 & 1.00 \\
\hline 6 to $<8$ & $0.95(0.92-0.98)$ & $1.09(1.05-1.12)$ & $1.08(1.04-1.11)$ \\
\hline 4 to $<6$ & $0.95(0.93-0.98)$ & $1.14(1.11-1.18)$ & $1.10(1.07-1.14)$ \\
\hline 0 to $<4$ & $1.07(1.04-1.10)$ & $1.23(1.19-1.27)$ & $1.13(1.10-1.17)$ \\
\hline \multicolumn{4}{|c|}{ Physical activity (mins/week) } \\
\hline $0+$ & 1.00 & 1.00 & 1.00 \\
\hline 1 to 149 & $1.75(1.61-1.90)$ & $1.44(1.33-1.57)$ & $1.29(1.19-1.41)$ \\
\hline 150 to 299 & $2.65(2.45-2.87)$ & $1.92(1.77-2.09)$ & $1.62(1.49-1.76)$ \\
\hline 300 to 539 & $3.35(3.09-3.62)$ & $2.35(2.17-2.55)$ & $1.86(1.71-2.02)$ \\
\hline$\geq 540$ & $4.05(3.75-4.38)$ & $3.06(2.82-3.31)$ & $2.30(2.12-2.49)$ \\
\hline
\end{tabular}

tReference category.

${ }^{a}$ Model 1. Odds for sitting time and moderate to vigorous physical activity, mutually adjusted for each other.

${ }^{b}$ Model 2. Same adjustment from model 1, additional adjustment for categories of age, household income, educational qualification, smoking status, marital status, weight status, sex, and remoteness and economic advantage of residential area.

'Model 3. Same adjustment from model 3, additional adjustment for categories of functional limitation and number of chronic diseases.

hours per day of sitting. The most physically active group was nearly three times as likely to report excellent health compared to the least active group (AOR for very highly active and sitting 0 to $<4$ hours per day versus zero minutes and sitting $\geq 8$ hours per day $=2.81 ; 95 \%$ $\mathrm{CI}=2.33,3.38, P_{\text {trend }}<0.001$ ).

Figure 2 depicts the fully adjusted odds (model 3 ) of excellent quality of life by combinations of physical activity and sitting time. The most physically active group was nearly three times as likely to report excellent quality of life compared to the least active group (AOR for very highly active and sitting 0 to $<4$ hours per day, versus zero minutes of physical activity and sitting $\geq 8$ hours per day $=2.90 ; 95 \% \mathrm{CI}=2.52,3.34, P_{\text {trend }}<0.001$ ).

\section{Discussion}

Stemming from a salutogenic approach and positive health message framework, we sought to investigate whether higher levels of physical activity and lower levels of sitting time were positively associated with excellent health and quality of life. This study's main finding was that both physical activity and sitting time were independently associated with excellent health and excellent quality of life, showing physical activity was the stronger influence of the two. These associations were attenuated when controlling for key demographic influences of age, household income, education, and weight status, but remained statistically significant and likely of public health significance. The associations were further attenuated when controlling for key health-related influences of chronic disease and physical limitation, but remained significant and likely meaningful with regard to the health of this population. Although there was some indication of interaction between physical activity and sitting time, the interactions were not statistically significant in fully adjusted models. Therefore, the final models for 


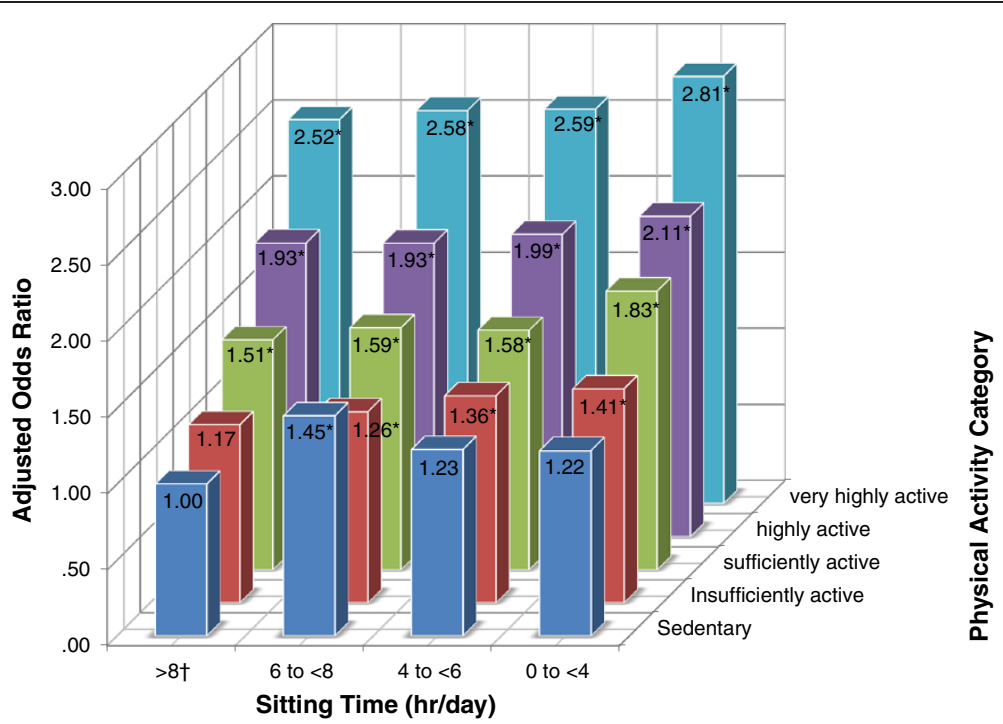

Figure 1 Odds of excellent self-rated health by category of sitting time and physical activity level. $N=194,545$. tReference category is those with lowest physical activity level who sit 8 or more hours per day. "Model adjusted for categories of age, household income, educational qualification, smoking status, marital status, weight status, sex, and remoteness and economic advantage of residential area, functional limitation, and number of chronic diseases. ${ }^{*} p<0.05 ;$ All AOR $>1.25$ Significantly different from 1.00 .

both excellent health and quality of life appear to reflect two independent main effects of physical activity and sitting time.

The likelihood of reporting excellent health and quality of life was substantially higher for individuals with an active lifestyle, as compared to their less active counterparts. Physical activity and sitting time both showed dose-response patterns of influence on the odds of excellent health and quality of life, when controlling for other key behavioral and environmental influences. In our stratified analyses, the robustness of relationships between the health-related outcomes and physical activity and sitting time was remarkable. Across most demographic strata, lower levels of sitting time generally showed small positive relationships with the aspirational outcomes of excellent health and quality of life. Physical

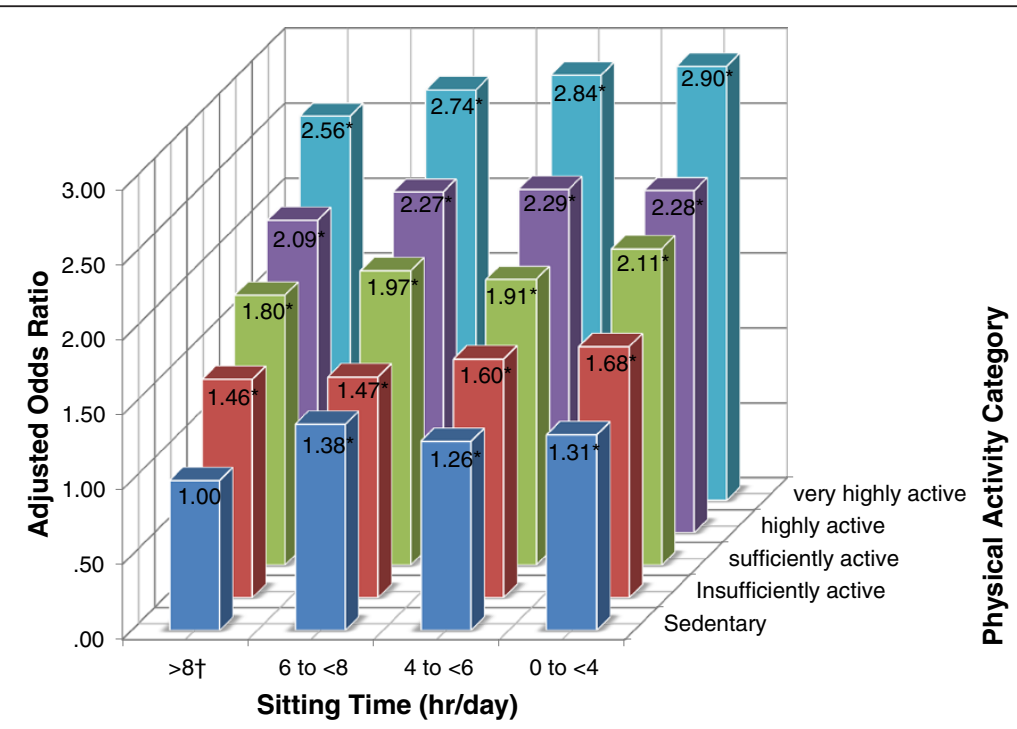

Figure 2 Odds of excellent quality of life by category of sitting time and physical activity level. $N=194,545$. theference category is those with lowest physical activity level who sit 8 or more hours per day. "Model adjusted for categories of age, household income, educational qualification, smoking status, marital status, weight status, sex, and remoteness and economic advantage of residential area, functional limitation, and number of chronic diseases. ${ }^{*} p<0.05$; All AOR $>1.25$ Significantly different from 1.00. 
activity too, was mostly consistent across strata, manifesting a strong salutogenic factor in this study. These patterns did not hold, however, for adults aged 85 and older $(n=4,230)$, or for underweight adults $(n=2,404)$ who constituted a small minority of this sample, and who have different profiles of health, quality of life, and risk of chronic disease than the majority of the population.

Conceptually, self-rated health reflects a global assessment related to functioning and presence or absence of diseases or symptoms, while health-related quality of life reflects the discrepancy between actual and desired functional status, and the overall impact of health on wellbeing [24]. Although the proportion of our sample reporting excellent quality of life was about $9 \%$ higher than the proportion reporting excellent health, the associations between lifestyle behaviors and these two health-related outcomes were strikingly similar. For most analyses, participants who were highly active or very highly active (300 minutes or more per week) were about twice as likely to report excellent health and excellent quality of life, as compared to their least active counterparts. Within the highly active or very highly active categories, the likelihood of excellent health or quality of life was higher for those reporting less sitting time per day, on the order of about a $20-30 \%$ difference between those sitting most and those sitting least each day at this level of physical activity. The influence of sitting, however, was clearly less of an influence than physical activity for likelihood of excellent health and quality of life.

\section{Integration with previous studies}

The present study's results are similar to those of Davies et al. [18], who examined associations between physical activity and screen time on health-related quality of life. Their study found that the combination of no physical activity and high screen time (e.g., watching televisiontypically sedentary behavior) was related to poorer quality of life in their sample of Australian adults. Our study differed from theirs, however, by focusing on excellent health and quality of life in a larger sample of middleaged and older adults, and examining sitting time rather than screen time. We also were able to statistically adjust for additional influences such as functional limitation, residential economic advantage and remoteness.

Kerr et al. [35] found that higher physical activity levels were significantly related to greater self-rated health in a sample of American adults, aged 66 years and up, but these researchers did not include sitting time in their analyses. Vallance et al. [19], however, recently showed a relationship between sitting and healthrelated quality of life in men aged 55 years and up, when adjusting for physical activity. In their study, those who reported sitting the least time had better physical, mental, and global health, compared to those who sat the most, but these relationships only held for weekend sitting time.

The present study aligns with previous studies showing that sitting time and physical activity are independently related to health-related outcomes when both lifestyle components are studied concurrently $[8-10,15]$. Similar to previous studies, our results showed some indication that the influence of physical activity and sitting time may interact $[7,10,18]$, but the interaction was not robust, and was attenuated and insignificant in the fully adjusted model.

The present findings suggest that physical activity and sitting time may each present a potential avenue to increase the likelihood of excellent health and quality of life, but that the combination of more physical activity and less sitting time may offer the greatest potential for attaining excellent health and quality of life. If such findings are borne out by additional research studies, positive health messages could be used to motivate populations to adopt more active lifestyles.

Although our findings align with much of the previous literature showing better health associated with more physical activity and less sitting time, not all studies have supported such relationships. In particular, Herber-Gast et al. [36] recently reported no association between sitting time and incidence of cardiovascular disease when controlling for physical activity and other relevant demographic and lifestyle factors in a longitudinal analysis of middle-aged Australian women. These authors found no interaction between physical activity and sitting time related to cardiovascular disease. The divergence from our findings may be due to differences in focusing on disease more than health, focusing solely on women, or possibly in differing measures of physical activity and sitting time.

In work more relevant to the salutogenic model, Vallance et al. [19] similarly did not show an association between sitting time and physical health, mental health, or global health when examining weekday sitting separately from weekend sitting. Trinh and colleagues [37] also reported few significant associations between sitting time and quality of life in cancer survivors. Södergren and colleagues [3] reported that although leisure time physical activity was associated with good health in adults aged 55-65 years, sitting time was not significantly related to measures of good health. The lack of association in these previous studies may represent unknown moderation by demographic variables, or may stem from residual confounding by physical activity level, age, work type and status, or other relevant influences on sitting time and health. Particularly relevant to our study, the impact of physical activity was stronger than that of sitting time, and controlling for this key 
lifestyle variable along with other important potential confounding variables in smaller datasets with more limited power or greater variability may partially explain null findings in previous studies [3]. Our observed fully adjusted odds ratios for sitting time were small in magnitude for the lowest versus highest sitting categories, and this magnitude of association is unlikely to be statistically significant in much smaller datasets.

\section{Areas for further study}

Despite the wealth of research on physical activity and health-related outcomes, the investigation of sitting time has only recently proliferated, and most of this work has focused on associations with chronic disease and mortality. Both physical activity and sitting time are linked with a wide range of health-related outcomes [5,38]. Both of these active lifestyle components can change energy expenditure, which is related to reduced risk of morbidity and mortality in much of the literature $[5,38]$. Yet, like the wide variety of biological and psychological mechanisms thought to play a role for physical activity, there is likely more to sitting's influence on health and quality of life than energy expenditure alone.

Spending long periods in occupational sitting is associated with overall fatigue, musculoskeletal pain, and poor health in data from interviews with office workers [39]. In the ergonomics literature, sitting is linked to one of the most prevalent chronic conditions, low back pain [40], frequently associated with disability [41]. Thus, prolonged bouts of sitting daily may potentially feature prominently in a downward spiral of decreased mobility, physical function, physical fitness, engagement with life, physical activity, and eventually greater risk of chronic disease [42], but much more work is required to examine these possibilities and their temporal sequence.

Beyond the focus on chronic diseases [15], indices of cardiometabolic health [12], and mortality [9], research on active lifestyles stemming from being physically active and limiting prolonged sitting has been moving into studies of mental wellbeing [43-45], cognitive function [38] and health-related quality of life $[3,19]$. More work is required in this area to determine associations with mood, energy level, sexual and neurological functioning, sleep, and activities of daily living, among many important health-related outcomes.

Further prospective studies should investigate active lifestyles and successful aging from a salutogenic approach [22], with a deliberate focus on the components of physical and cognitive functioning and engagement with life, plus avoidance of disease [21]. More evidence from longitudinal prospective research is needed, including the continued use of The 45 and Up Study data as further time points become available. Armed with epidemiological evidence, further work in etiology and lifestyle determinants of successful aging can include experimental studies, and public health intervention work can examine positive message framing to promote excellent health and quality of life through more active lifestyles. Public health efforts should also address a need for guidelines or recommendations for limiting prolonged sitting or reductions in this sedentary behavior, to accompany physical activity guidelines.

\section{Strengths and limitations}

A major strength of the current study is the use of a very large heterogeneous sample of Australian adults, with a diversity of age, socioeconomic status, residential characteristics, and other demographic and lifestyle influences on health and quality of life. An additional strength of this study was our ability to stratify analyses and statistically control for potential confounding variables that may limit or distort findings from such observational studies. Lastly, our study was novel in the use of a salutogenic approach to frame positive health messages toward motivating healthful active lifestyle behaviors and successful aging.

Opposite these strengths stand the major limitation of cross-sectional analysis of these baseline data from the ongoing longitudinal 45 and Up Study. Cross-sectional analysis precludes any causal implications of the observed relationships. Further studies with this 45 and Up Study and others will be better able to show temporality of association, particularly as follow-up data become available in the near future. Self-report measures of sitting time and physical activity may be susceptible to various types of bias, but our measures of physical activity and sitting time were sufficient for ranking individuals within an epidemiological dataset for analysis [31]. Also, our measures of overall health and quality of life were self-reported, and therefore inherently subjective. Despite this, patient-reported health status has been shown to be an independent predictor of subsequent mortality, cardiovascular events, hospitalization, and costs of care [24]. Therefore, such measures are highly relevant in public health research.

\section{Conclusions}

Physical activity and sitting time are independently associated with excellent health and quality of life in this large diverse sample of Australian middle-aged and older adults. The present study's findings bolster evidence to inform efforts to increase moderate-to-vigorous physical activity and decrease time spent sitting toward the achievement of better population health and pursuit of successful aging. Public health efforts can use the accumulating body of evidence on active lifestyles to develop guidelines or recommendations for sitting, in addition to those of physical activity. Public health efforts could 
consider framing positive health messages and health promotion interventions aimed at achievement of higher levels of health and quality of life through moving more and sitting less to motivate middle-aged and older adults to improve their lifestyles.

\section{Additional file}

Additional file 1: Table S1. Age-stratified odds of excellent overall health and quality of life by sitting time and physical activity $(N=194,545)$ Table S2. Sex-stratified and ancestry-stratified odds of excellent overall health and quality of life by sitting time and physical activity $(N=194,545)$. Table S3. Household-income-stratified odds of excellent overall health and quality of life by sitting time and physical activity $(N=194,545)$. Table S4. Body-massindex-stratified odds of excellent overall health and quality of life by sitting time and physical activity $(\mathrm{N}=194,545)$

\section{Abbreviations}

OR: Odds ratio; AOR: Adjusted odds ratio; Cl: Confidence interval; ExH: Excellent self-rated health; ExQoL: Excellent self-rated quality of life; BMI: Body mass index; NSW: New South Wales; UWS: University of Western Sydney; AAS: Active Australia Survey; WHO: World Health Organization; MOSPF: Medical outcomes study physical functioning scale; IPAQ: International physical activity questionnaire; SD: Standard deviation.

\section{Competing interests}

The authors declare that they have no competing interests.

\section{Authors' contributions}

RRR conceived of the study, participated in the design and coordination of the study, performed the statistical analysis and drafted the manuscript. SKR participated in the design and coordination of the study, assisted with the statistical analysis and contributed to the preparation of the manuscript. MJD participated in the design and coordination of the study, assisted with statistical analysis and contributed to the preparation of the manuscript. GSK participated in the design and coordination of the study and contributed to the preparation of the manuscript. All authors read and approved the final manuscript.

\section{Acknowledgements}

The Sax Institute manages The 45 and Up Study in collaboration with major partner Cancer Council New South Wales and partners the National Heart Foundation of Australia (NSW Division); NSW Ministry of Health; beyondblue: the national depression and anxiety initiative; Ageing, Disability and Home Care, Department of Family and Community Services NSW; UnitingCare Ageing; and the Australian Red Cross Blood Service. MJD is in part supported by the CQUniversity Health CRN. Publication of this article was funded in part by the Kansas State University Open Access Publishing Fund.

\section{Author details}

${ }^{1}$ School of Science and Health, University of Western Sydney, Sydney, Australia. ${ }^{2}$ Department of Human Nutrition, Kansas State University, Manhattan, KS, USA. ${ }^{3}$ Institute for Health and Social Science Research, Centre for Physical Activity Studies, Central Queensland University, Rockhampton, Australia.

\section{Received: 21 October 2013 Accepted: 23 October 2013}

Published: 13 November 2013

\section{References}

1. World Health Organization: Good health adds life to years. Global brief for World Health Day 2012; 2012:28. http://whqlibdoc.who.int/hq/2012/ WHO_DCO_WHD_2012.2_eng.pdf.

2. Martinez-Gómez D, Guallar-Castillón P, León-Muñoz LM, López-Garcia E, Rodríguez-Artalejo F: Combined impact of traditional and non-traditional health behaviors on mortality: a national prospective cohort study in Spanish older adults. BMC Med 2013, 11:47.

3. Södergren M, McNaughton SA, Salmon J, Ball K, Crawford DA: Associations between fruit and vegetable intake, leisure-time physical activity, sitting time and self-rated health among older adults: cross-sectional data from the WELL study. BMC Public Health 2012, 12:551.

4. Owen N, Healy GN, Matthews CE, Dunstan DW: Too much sitting: the populationhealth science of sedentary behavior. Exerc Sport Sci Rev 2010, 38:105.

5. US Department of Health and Human Services: 2008 physical activity guidelines for Americans: Be active, healthy and happy. Washington DC: US Department of Health and Human Services; 2008.

6. León-Muñoz LM, Martínez-Gómez D, Balboa-Castillo T, López-García E, Guallar-Castillón P, Rodríguez-Artalejo F: Continued sedentariness, change in sitting time, and mortality in older adults. Med Sci Sports Exerc 2013. Epub ahead of print.

7. Pavey TG, Peeters GMEE, Brown WJ: Sitting-time and 9-year all-cause mortality in older women. Br J Sports Med 2012. Epub ahead of print.

8. Katzmarzyk PT, Church TS, Craig CL, Bouchard C: Sitting time and mortality from all causes, cardiovascular disease, and cancer. Med Sci Sports Exerc 2009, 41:998-1005.

9. van der Ploeg HP, Chey T, Korda RJ, Banks E, Bauman A: Sitting time and all-cause mortality risk in 222497 Australian adults. Arch Intern Med 2012, 172:494-500.

10. Patel AV, Bernstein L, Deka A, Spencer Feigelson H, Campbell PT, Gapstur SM, Colditz GA, Thun MJ: Leisure time spent sitting in relation to total mortality in a prospective cohort of US adults. Am J Epidemiol 2010, 172:419-429.

11. Dunstan DW, Salmon J, Owen N, Armstrong T, Zimmet PZ, Welborn TA, Cameron AJ, Dwyer T, Jolley D, Shaw JE: Associations of TV viewing and physical activity with the metabolic syndrome in Australian adults. Diabetologia 2005, 48:2254-2261.

12. Healy GN, Wijndaele K, Dunstan DW, Shaw JE, Salmon J, Zimmet PZ, Owen $\mathrm{N}$ : Objectively measured sedentary time, physical activity, and metabolic risk: The Australian Diabetes, Obesity and Lifestyle Study (AusDiab). Diabetes Care 2008, 31:369-371.

13. Hu FB, Leitzmann MF, Stampfer MJ, Colditz GA, Willett WC, Rimm EB: Physical activity and television watching in relation to risk for type 2 diabetes mellitus in men. Arch Intern Med 2001, 161:1542-1548.

14. Hu FB, Li TY, Colditz GA, Willett WC, Manson JE: Television watching and other sedentary behaviors in relation to risk of obesity and type 2 diabetes mellitus in women. JAMA 2003, 289:1785-1791.

15. George ES, Rosenkranz RR, Kolt GS: Chronic disease and sitting time in middle-aged Australian males: findings from the 45 and Up Study. Int J Behav Nutr Phys Act 2013, 10:20.

16. Patel AV, Rodriguez C, Pavluck AL, Thun MJ, Calle EE: Recreational physical activity and sedentary behavior in relation to ovarian cancer risk in a large cohort of US women. Am J Epidemiol 2006, 163:709-716.

17. Peeters GMEE, Burton NW, Brown WJ: Associations between sitting time and a range of symptoms in mid-age women. Prev Med 2013, 56:135-141.

18. Davies CA, Vandelanotte C, Duncan MJ, van Uffelen JG: Associations of physical activity and screen-time on health related quality of life in adults. Prev Med 2012, 55:46-49.

19. Vallance JK, Eurich D, Marshall AL, Lavallee CM, Johnson ST: Associations between sitting time and health-related quality of life among older men. Mental Health Phys Act 2013, 6:49-54.

20. Garatachea N, Molinero O, Martinez-Garcia R, Jiménez-Jiménez R, GonzálezGallego J, Márquez S: Feelings of well being in elderly people: relationship to physical activity and physical function. Arch Gerontol Geriatr 2009, 48:306-312

21. Rowe JW, Kahn RL: Successful aging. Gerontologist 1997, 37:433-440.

22. Antonovsky A: The salutogenic model as a theory to guide health promotion. Health Promot Int 1996, 11:11-18.

23. Gallagher KM, Updegraph JA: Health message framing effects on attitudes, intentions, and behavior: a meta-analytic review. Ann Behav Med 2012, 43:101-116.

24. Rumsfeld JS, Alexander KP, Goff DC Jr, Graham MM, Ho PM, Masoudi FA, Moser DK, Roger VL, Slaughter MS, Smolderen KG, Spertus JA, Sullivan MD, TreatJacobson D, Zerwic JJ, on behalf of the American Heart Association Council on Quality of Care and Outcomes Research, Council on Cardiovascular and Stroke Nursing, Council on Epidemiology and Prevention, Council on Peripheral Vascular Disease, and Stroke Council: Cardiovascular health: the importance of measuring patient-reported health status: a scientific statement from the American Heart Association. Circulation 2013, 127:2233-2249.

25. 45 and Up Study Collaborators: Cohort profile: The 45 and Up Study. Int J Epidemiol 2008, 35:941-947. 
26. The Sax Institute: The 45 and Up Study website. https://www.saxinstitute.org. au/our-work/45-up-study/.

27. Australian Institute of Health and Welfare (AlHW): The Active Australia Survey: a guide and manual for implementation, analysis, and reporting. Canberra: AlHW; 2003

28. Brown WJ, Trost SG, Bauman A, Mummery K, Owen N: Test-retest reliability of four physical activity measures used in population surveys. J Sci Med Sport 2004, 7:205-215.

29. Heesch KC, Hill RL, Van Uffelen JG, Brown WJ: Are Active Australia physical activity questions valid for older adults? J Sci Med Sport 2011, 14:233-237.

30. Craig CL, Marshall AL, Sjöström M, Bauman AE, Booth ML, Ainsworth BE, Pratt M, Ekelund U, Yngve A, Sallis JF: International Physical Activity Questionnaire: 12-country reliability and validity. Med Sci Sports Exerc 2003, 35:1381-1395.

31. Atkin AJ, Gorely T, Clemes SA, Yates T, Edwardson C, Brage S, Salmon J, Marshall SJ, Biddle SJ: Methods of measurement in epidemiology: Sedentary behaviour. Int J Epidemiol 2012, 41:1460-1471.

32. World Health Organization: World Health Organization - Global database on body mass index. http://apps.who.int/bmi/index.jsp?introPage=intro_1.html.

33. Stewart A, Kamberg CJ: Physical functioning measures. In Measuring functioning and well-being: The Medical Outcomes Study approach. Edited by Stewart A, Ware JE. Durham, North Carolina: Duke University Press; 1992.

34. Haley SM, McHorney CA, Ware JJE: Evaluation of the MOS SF-36 physical functioning scale (PF-10): I. Unidimensionality and reproducibility of the Rasch Item scale. J Clin Epidemiol 1994, 47:671-684.

35. Kerr J, Sallis JF, Saelens BE, Cain K, Conway TL, Frank LD, King AC: Outdoor physical activity and self rated health in older adults living in two regions of the U.S. Int J Behav Nutr Phys Act 2012, 9:89.

36. Herber-Gast GC, Jackson CA, Mishra GD, Brown WJ: Self-reported sitting time is not associated with incidence of cardiovascular disease in a population-based cohort of mid-aged women. Int J Behav Nutr Phys Act 2013, 10:55

37. Trinh L, Plotnikoff RC, Rhodes RE, North S, Courneya KS: Associations between sitting time and quality of life in a population-based sample of kidney cancer survivors. Mental Health Phys Act 2013, 6:16-23.

38. New Zealand Ministry of Health: A literature review of evidence on physical activity for older people and a review of existing physical activity guidelines for older people. Wellington; 2011.

39. Gilson ND, Burton NW, Van Uffelen JG, Brown WJ: Occupational sitting time: employees' perceptions of health risks and intervention strategies. Health Promot J Austr 2011, 22:38-43.

40. Magnusson ML, Pope $\mathrm{MH}$ : A review of the biomechanics and epidemiology of working postures. J Sound Vib 1998, 215:965-976.

41. Walker BF, Muller R, Grant WD: Low back pain in Australian adults Prevalence and associated disability. J Manipulative Physiol Ther 2004 27:238-244.

42. Yorston LC, Kolt GS, Rosenkranz RR: Physical activity and physical function in older adults: the 45 and up study. J Am Geriatr Soc 2012, 60:719-725.

43. Faulkner G, Biddle SJH: Standing on top of the world: Is sedentary behavior associated with mental health? Mental Health Phys Act 2013, 6:1-2.

44. Lampinen P, Heikkinen RL, Kauppinen M, Heikkinen E: Activity as a predictor of mental well-being among older adults. Aging Ment Health 2006, 10:454-466.

45. Fox KR, Stathi A, McKenna J, Davis MG: Physical activity and mental wellbeing in older people participating in the Better Ageing Project. Eur Appl Physiol 2007, 100:591-602.

doi:10.1186/1471-2458-13-1071

Cite this article as: Rosenkranz et al: Active lifestyles related to excellent self-rated health and quality of life: cross sectional findings from 194,545 participants in The 45 and Up Study. BMC Public Health 2013 13:1071.

\section{Submit your next manuscript to BioMed Central and take full advantage of:}

- Convenient online submission

- Thorough peer review

- No space constraints or color figure charges

- Immediate publication on acceptance

- Inclusion in PubMed, CAS, Scopus and Google Scholar

- Research which is freely available for redistribution 